\title{
Cyclin-dependent kinase 10 prevents glioma metastasis via modulation of Snail expression
}

\author{
HUI LI ${ }^{1}$, YANJIE YOU ${ }^{2}$ and JIANFENG LIU ${ }^{1}$ \\ ${ }^{1}$ Department of Neurosurgery, The First Hospital of Hebei Medical University, Shijiazhuang, Hebei 050000; \\ ${ }^{2}$ Department of Gastroenterology, Ningxia Hui Autonomous Region People's Hospital, Yinchuan, \\ Ningxia Hui Autonomous Region 750021, P.R. China
}

Received August 18, 2017; Accepted May 4, 2018

DOI: $10.3892 / \mathrm{mmr} .2018 .9059$

\begin{abstract}
Cyclin-dependent kinase 10 (CDK10) has been indicated to be a candidate tumor suppressor in multiple cancer types. However, to the best of the authors' knowledge, its biological and regulatory functions in glioma have not been previously reported. In the present study, it was demonstrated that overexpression of CDK10 inhibited glioma cell proliferation and metastasis. By contrast, knockdown of CDK10 expression promoted these malignant phenotypes. It was additionally indicated that dysregulated CDK10 expression was associated with epithelial-mesenchymal transition (EMT) and that it regulated the expression of zinc finger protein SNAI1 (Snail). Furthermore, silencing Snail expression rescued EMT phenotypes induced by CDK10 knockdown, suggesting that Snail may be involved in the mechanistic association between CDK10 and EMT. The present study illustrated that downregulation of CDK10 expression activated Snail-driven EMT and consequently promoted glioma metastasis, suggesting that CDK10 may serve as a potential molecular target for glioma therapy.
\end{abstract}

\section{Introduction}

Glioma is the most common type of brain tumor and is responsible for $\sim 70 \%$ of malignancies of the central nervous system worldwide (1-3). Evidence suggests that patients with glioblastoma, the most aggressive subtype of glioma, exhibit a median survival period of 14 months. Regardless of advances in the use of surgical treatment in combination with radiation and chemotherapy, the prognosis of patients with glioma remains poor, largely due to diffuse tumor cell invasion into the surrounding normal brain tissue $(3,4)$. In addition, migratory

Correspondence to: Professor Jianfeng Liu, Department of Neurosurgery, The First Hospital of Hebei Medical University, 89 Donggang Road, Shijiazhuang, Hebei 050000, P.R. China E-mail: 18639563018@163.com

Key words: glioma, cyclin-dependent kinase 10, metastasis, epithelial-mesenchymal transition, zinc finger protein SNAI1 glioma cells may develop resistance to clinical therapies, further confounding efforts to eradicate them. To date, the molecular mechanism of glioma metastasis has remained unclear and data from previous studies are difficult to interpret due to the complexity of the malignancy. Therefore, the identification of specific genes, which may serve crucial roles during the metastasis and progression of glioma, is urgently required (5).

Cyclin-dependent kinase 10 (CDK10), also termed PISSLRE, is a CDK1-associated kinase, which is important in cell cycle progression from the $\mathrm{G} 2$ to the $\mathrm{M}$ phase (6-8). It has been suggested that CDK10 may serve as a key determinant for endocrine therapeutic resistance in breast cancer (9). Furthermore, silencing of CDK10 expression reduces the dependence of breast cancer cells on estrogen (9). It has been demonstrated that CDK10 expression is downregulated in nasopharyngeal carcinoma, and that overexpression of CDK10 strongly suppresses the growth and metastasis of cancer cells (10), which is consistent with studies on hepatocellular carcinoma and biliary tract cancer $(11,12)$. More recent reports revealed that CDK10 expression levels were decreased in breast cancer, and that CDK10 expression was significantly associated with lymph node metastasis and unfavorable patient survival $(13,14)$. These data indicated that CDK10 may serve as a tumor suppressor and that silencing of CDK10 may contribute to metastatic progression; however, the precise underlying molecular mechanism is largely unknown. In the present study, data indicating a novel molecular mechanism by which CDK10 may prevent the metastatic progression of glioma were presented.

\section{Materials and methods}

Cell culture. The human glioma cell line U251-MG was donated by Miss Jiongyu Chen (Cancer Hospital of Shantou University Medical College, Shantou, China) and cultured in Dulbecco's modified Eagle's medium (DMEM; Gibco; Thermo Fisher Scientific, Inc., Waltham, MA, USA) supplemented with $10 \%$ fetal bovine serum (FBS; Gibco; Thermo Fisher Scientific, Inc.) at $37^{\circ} \mathrm{C}$ in $5 \% \mathrm{CO}_{2}$.

Gene over-expression and RNA interference. A recombinant CDK10-expressing plasmid (pcDNA3.1-CDK10) and 
pre-designed and validated small interfering RNAs (siRNAs) targeting CDK10 (5'-GUCCCAGUAAAGCCAAUGATT-3' and 5'-UCAUUGGCUUUACUGGGACTT3') and Snail (5'-GACUCUAAUCCAGAGUUUATT-3' and 5'-UAAACU CUGGAUUAGAGUCTT-3') were obtained from Guangzhou RiboBio Co., Ltd. (Guangzhou, China) and prepared as described previously (10). Scramble siRNA (5'-GUACGCCAA AAGUUAAACC-3' and 5'-CAUGCGGUUUUCAAUUUGG) was also prepared as a control. Cells were seeded at $1 \times 10^{5}$ cells per well in 6-well plates. Cell transfection was conducted using Lipofectamine 2000 (Invitrogen; Thermo Fisher Scientific, Inc.), according to the manufacturer's protocol. Cells were harvested at $48 \mathrm{~h}$ post-transfection for use in the subsequent experiments.

Cell viability assay. MTT colorimetric assays were performed to measure the growth rate of cancer cells, as described previously $(10,15,16)$. In brief, cells were placed in 96-well plates at a density of $4 \times 10^{3}$ cells/well and incubated for $72 \mathrm{~h}$. Formazan crystals were dissolved in dimethyl sulfoxide and optical density was measured at $490 \mathrm{~nm}$. A scrambled siRNA-transfected group and an empty vector-transfected group served as controls for knockdown and overexpression of CDK10, respectively. All experiments were conducted with six replicates and repeated three times independently.

Colony formation assay. Soft agar colony formation assays were performed as previously described $(10,16)$. Cells were suspended in DMEM containing $0.35 \%$ agar, $10 \%$ FBS and 800 mg/l G418 (Gibco; Thermo Fisher Scientific, Inc.) at a density of $5 \times 10^{3}$ cells/well, in 6-well plates containing DMEM supplemented with $0.5 \%$ agar, $10 \%$ FBS and $800 \mathrm{mg} / 1$ G418. Following a 15 -day incubation at $37^{\circ} \mathrm{C}$, the number of colonies was counted using a light microscope (magnification, x100; Carl Zeiss AG, Oberkochen, Germany) and the images were captured. A cluster containing $\geq 50$ cells was defined as a colony. Each experiment was performed in triplicate and repeated three times.

Wound healing assay. This assay was performed according to previously described standard methods (17). In brief, cells were cultured in 6-well plates to $80-90 \%$ confluence. The cell layer was wounded using a $200-\mu 1$ pipette tip, washed twice in PBS and cultured in medium containing $2 \% \mathrm{FBS}$ at $37^{\circ} \mathrm{C}$. The wound sizes were measured under a under a light microscope as above at 0 and $24 \mathrm{~h}$ following wounding. The area of migrating cells was estimated by counting the number of pixels after the images had been converted to Photoshop data (Adobe Systems, Inc., San Jose, CA, USA). Relative distance migrated was measured as the migration of CDK10-knockdown group or overexpression group divided by that of the control group.

Invasion assay. Cell invasion was measured using 24-well Transwell chambers (Corning Costar; Corning Incorporated, Corning, NY, USA). Matrigel (BD Biosciences, Franklin Lakes, NJ, USA) was diluted 1:8 with cold serum-free DMEM and coated onto the Transwell inserts. Cells were plated into the upper chambers at $2 \times 10^{4}$ cells per well in $100 \mu \mathrm{l}$ serum-free DMEM. The bottom chamber was filled with DMEM containing $10 \%$ FBS. Following incubation at $37^{\circ} \mathrm{C}$ for
$24 \mathrm{~h}$, non-invasive cells in the upper chamber were removed. Invasive cells on the lower surface of the inserts were fixed with $4 \%$ paraformaldehyde at room temperature for $30 \mathrm{~min}$, stained with $0.2 \%$ crystal violet at room temperature for $2 \mathrm{~h}$ and counted under a light microscope (magnification, x100).

Western blot analysis. Western blot analysis was performed as previously described $(10,13,15,16)$. A total of $50 \mu$ g proteins were separated by $12 \%$ SDS-PAGE and transferred to a polyvinylidene difluoride membrane (EMD Millipore, Billerica, MA, USA). The membranes were incubated in blocking buffer (Tris-buffered saline with $0.1 \%$ Tween and 5\% nonfat dry milk) at $37^{\circ} \mathrm{C}$ for $1 \mathrm{~h}$, and subsequently incubated with the following antibodies overnight at $4^{\circ} \mathrm{C}$ : Rabbit polyclonal antibodies against vimentin (cat. no. ab45939; 1:500; Abcam, Cambridge, MA, USA), neural (N)-cadherin (cat. no. ab18203; 1:1,000; Abcam), twist-related protein 1 (Twist; cat. no. ab50581; 1:500; Abcam), epithelial (E)-cadherin (cat. no. sc-7870; 1:500; Santa Cruz Biotechnology, Inc., Dallas, TX, USA), occludin (cat. no. sc-8144; 1:500; Santa Cruz Biotechnology, Inc.), Snail (cat. no. sc-10432; 1:500; Santa Cruz Biotechnology, Inc.), zinc finger protein SNAI2 (Slug; cat. no. sc-10437; 1:500; Santa Cruz Biotechnology, Inc.), zinc finger E-box-binding homeobox 1 (ZEB1; cat. no. sc-10432; 1:500; Santa Cruz Biotechnology, Inc.) and CDK10 (cat. no. ab50581; 1:500; Abcam). The membranes were subsequently incubated with a horseradish peroxidase-conjugated secondary goat antibody against rabbit immunoglobulin G (cat. no. sc-2004; 1:2,000; Santa Cruz Biotechnology, Inc.). Signals were visualized with the Enhanced Chemiluminescence (ECL) System kit following the protocols of the manufacturer (Amersham Pharmacia; GE Healthcare, Chicago, IL, USA). Blots were probed with an anti-GAPDH monoclonal antibody (cat. no. ab9485; 1:2,000; Abcam) was utilized to confirm equal loading of the different samples. Quantification of the intensity of protein expression levels in the immunoblot was conducted using Bio-Rad Quantity One software (version 4.5.2; Bio-Rad Laboratories, Inc., Hercules, CA, USA).

Statistical analysis. Statistical analyses were performed using SPSS software (version 17.0; SPSS, Inc., Chicago, IL, USA). Data are presented as the mean \pm standard deviation. One-way analysis of variance, followed by the Student-Newman-Keuls post-test for multiple comparisons, was used for comparisons of two or more groups. $\mathrm{P}<0.05$ was considered to indicate a statistically significant difference.

\section{Results}

CDK10-knockdown promotes glioma cell proliferation and invasion. CDK10 is characterized as a tumor suppressor and its expression has been demonstrated to be downregulated in various types of cancer (9-14). The effect of CDK10 on cell proliferation and metastasis was investigated by silencing CDK10 expression in glioma cells. An siRNA targeting CDK10 (si-CDK10) was transfected into U251-MG cells (Fig. 1A). A scrambled siRNA served as a control. U251-MG cells transfected with si-CDK10 exhibited a significant increase in cell proliferation compared with the control cells $(\mathrm{P}<0.01$; Fig. 1B). Furthermore, CDK10-knockdown in U251 
A
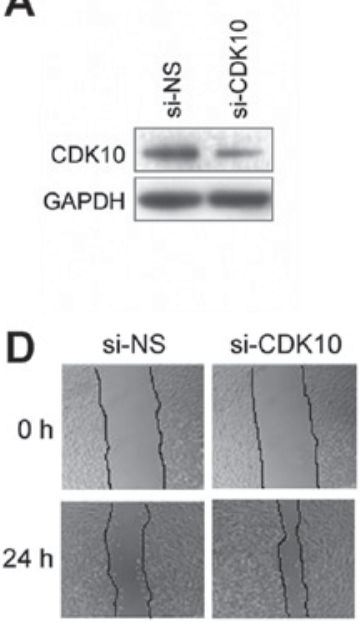
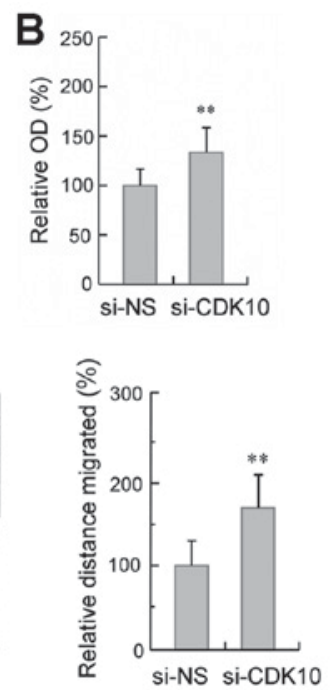

C
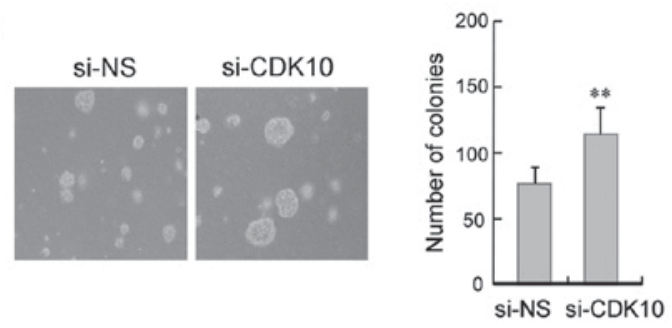

$\mathrm{E}$
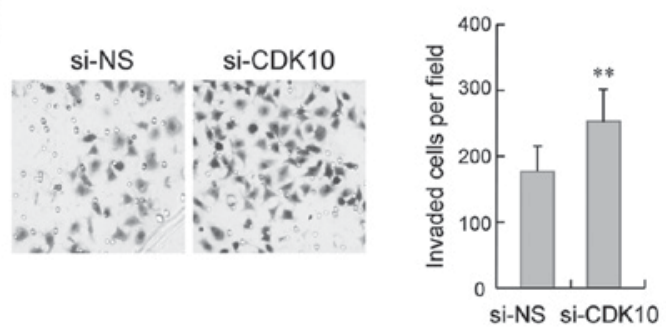

Figure 1. CDK10-knockdown promotes glioma cell proliferation, migration and invasion. (A) CDK10-knockdown in U251 cells was confirmed by western blot analysis. CDK10-knockdown promoted U251 cell proliferation, as determined by (B) MTT and (C) colony formation assays (magnification, x100). (D) CDK10-silencing promoted the migration of U251 cells in a wound-healing assay (magnification, $\mathrm{x} 100$ ). (E) CDK10-knockdown increased the invasiveness of U251 cells as demonstrated by a Transwell assay (magnification, $x 100$ ). Each experiment was repeated at least three times. ${ }^{* * *} \mathrm{P}<0.01 \mathrm{vs}$. si-NS. CDK, cyclin dependent kinase; si-NS, non-specific control siRNA; OD, optical density.

A

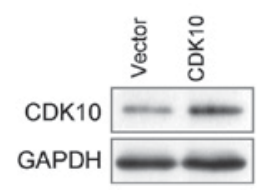

D

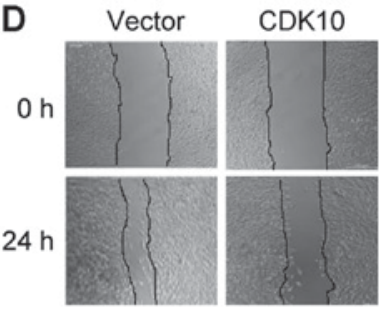

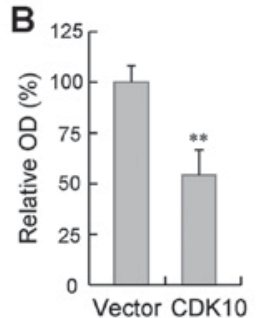

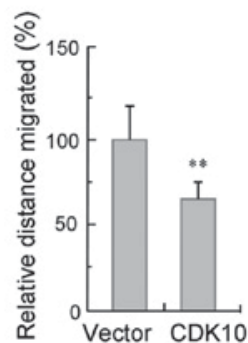

C
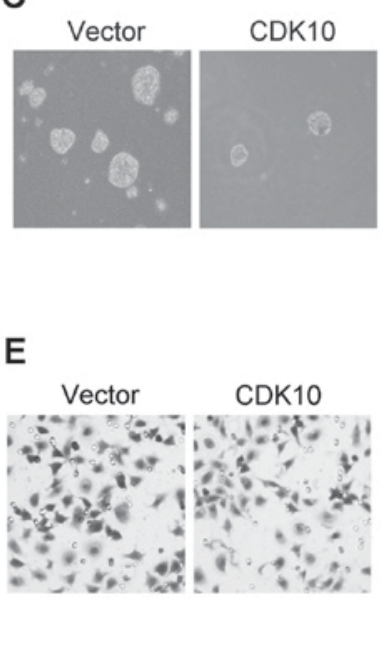
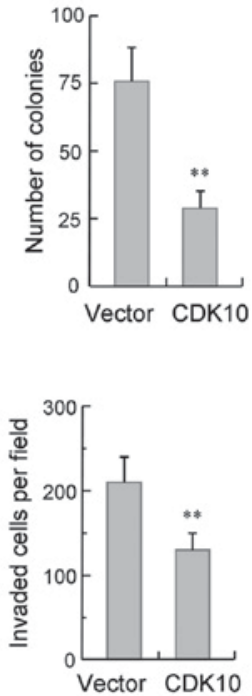

Figure 2. CDK10 overexpression inhibits glioma cell proliferation and metastasis. (A) CDK10 overexpression in U251 cells was indicated by western blotting. Overexpression of CDK10 inhibited U251 cell proliferation, as determined by (B) MTT and (C) colony formation assays (magnification, x100). (D) Ectopic CDK10 expression inhibited the migration of U251 cells, as determined using a wound-healing assay (magnification, x100). (E) Ectopic CDK10 expression decreased U251 cell invasive ability in a Transwell assay (magnification, $\mathrm{x} 100$ ). Each experiment was repeated at least three times. ${ }^{* * *} \mathrm{P}<0.01 \mathrm{vs}$. vector control. CDK, cyclin dependent kinase; OD, optical density.

cells led to significantly increased colony formation compared with the control cells $(\mathrm{P}<0.01$; Fig. 1C).

Wound-healing assays were performed to examine the effect of CDK10-knockdown on cell migration. CDK10-knockdown resulted in significantly enhanced wound closure compared with the control cells $(\mathrm{P}<0.01$; Fig. 1D). The anti-metastatic function of CDK10 was also examined using a Transwell assay. U251-MG cells with CDK10-knockdown exhibited a significant increase in the number of invasive cell numbers compared with control cells $(\mathrm{P}<0.01$; Fig. 1E). Together, these data indicated that CDK10-knockdown in glioma cells promoted cell proliferation, migration and invasion.
Overexpression of CDK10 inhibits glioma cell growth and invasion. Subsequently, the biological function of ectopic CDK10 expression was examined in glioma cells. Overexpression of CDK10 expression was confirmed by western blot analysis (Fig. 2A). As detected by MTT assays, the growth rates of U251 cells overexpressing CDK10 were significantly attenuated compared with control cells ( $\mathrm{P}<0.01$; Fig. 2B). This was further confirmed by a colony formation assay (Fig. 2C).

It was next determined whether CDK10 was able to affect the mobility of glioma cells. U251 cells overexpressing CDK10 exhibited significantly reduced wound closure compared with control cells ( $\mathrm{P}<0.01$; Fig. 2D). These results were supported by a Matrigel invasion assay, which demonstrated that U251 cells 


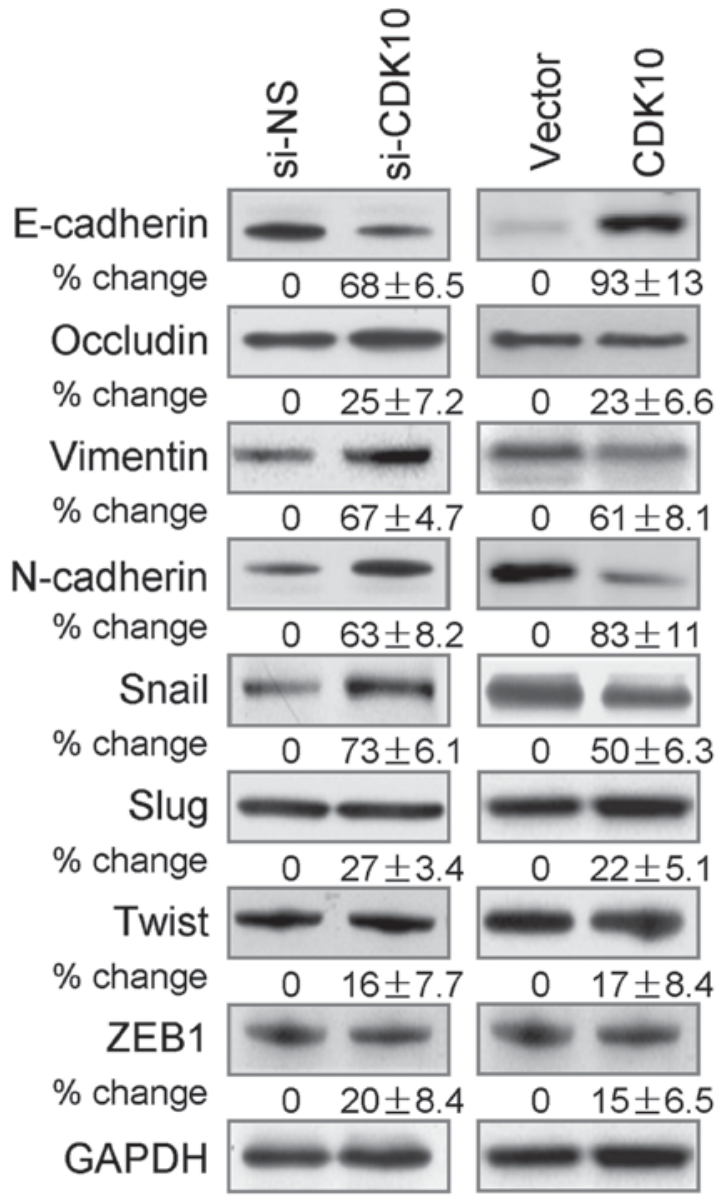

Figure 3. Western blot analysis of mesenchymal and epithelial markers. U251 cells were transfected with si-CDK10 or pcDNA-CDK10 and cultured for $48 \mathrm{~h}$, and the protein expression levels of mesenchymal and epithelial protein markers were examined by western blot analysis with the indicated antibodies. GAPDH was used as an internal loading control. CDK, cyclin dependent kinase; si, small interfering; E, epithelial; N, neural; si-NS, non-specific control siRNA; ZEB1, zinc finger E-box-binding homeobox 1; Twist, twist-related protein 1; Slug, zinc finger protein SNAI2; Snail, zinc finger protein SNAI1.

overexpressing CDK10 exhibited a significant decrease in cell invasiveness compared with control cells $(\mathrm{P}<0.01$; Fig. 2E). This suggested that overexpression of CDK10 suppressed the migration and invasion of glioma cells.

CDK10 regulates epithelial-mesenchymal transition (EMT)-associated proteins in glioma cells. It is well established that EMT serves a crucial role in the metastasis of various types of cancer. The shift to EMT-associated protein expression is regarded as an important mechanism in the promotion of motility in glioma cancer cells $(18,19)$. Therefore, the expression statuses of EMT protein markers were analyzed by western blot analysis. It was demonstrated that CDK10-knockdown in U251 cells caused downregulation of E-cadherin expression and upregulation of mesenchymal markers, vimentin and $\mathrm{N}$-cadherin (Fig. 3). The transcription factors, Snail, Slug, Twist and ZEB1 serve critical roles in modulating EMT $(20,21)$. Silencing of CDK10 resulted in increased Snail protein expression levels. However, no difference was observed with respect to ZEB1, Twist or Slug. Conversely, overexpression of CDK10 in U251 cells resulted in increased E-cadherin, and decreased vimentin and $\mathrm{N}$-cadherin protein expression levels (Fig. 3, right panel). Furthermore, overexpression of CDK10 impaired Snail protein expression levels. Notably, U251 cells continued to express occludin, despite CDK10 dysregulation.

Snail expression is crucial for CDK10-associated EMT in glioma cells. It was hypothesized that Snail may be critical for CDK10 regulation of EMT in glioma cells. U251 cells were co-transfected with CDK10-targeting and Snail-targeting siRNAs. The protein expression level of EMT-associated markers was detected by western blot analysis, and the migration and invasion capabilities were also assessed. Downregulation of E-cadherin protein expression following CDK10-silencing was partially rescued by Snail-knockdown (Fig. 4A). Similarly, Snail-knockdown reversed the upregulation of vimentin and $\mathrm{N}$-cadherin following CDK10-silencing. Additionally, CDK10-silencing resulted in increased invasion and migration abilities of U251 cells, while Snail-knockdown partially, although significantly, reversed these phenotypes $(\mathrm{P}<0.01$; Fig. 4B and $\mathrm{C})$. The success of the transfection with si-Snail was confirmed by western blot analysis (Fig. 4D). Collectively, these observations suggested that Snail expression may be required for the EMT and metastatic progression of glioma in which CDK10 is downregulated.

\section{Discussion}

To determine whether CDK10 acts as a tumor suppressor gene in glioma, gain- and loss-of-function assays were conducted to analyze the biological roles of CDK10 in U251 cells. It was demonstrated that knockdown of CDK10 induced EMT in glioma cells, due to downregulation of epithelial markers and upregulation of mesenchymal markers. Increased cell proliferation, migration and invasion were also observed following CDK1-knockdown. The opposite results were observed in CDK10-overexpressing cells.

Since it was demonstrated that CDK10 overexpression affected the metastasis of glioma, further experiments aimed to reveal the underlying mechanism. EMT is considered to be highly relevant to glioma metastasis, which occurs at the invasive front of glioma tissues (18). A previous study demonstrated that the expression status of EMT markers was closely associated with mesenchymal differentiation in gliosarcoma, a rare form of glioblastoma characterized by a biphasic mode of glial and mesenchymal differentiation (22). The present study demonstrated a mechanism through which CDK10 may regulate EMT. CDK10-knockdown by siRNA activated EMT and consequently increased the motility and invasiveness of glioma cells. Conversely, overexpression of CDK10 suppressed these malignant phenotypes. These data indicated that CDK10 was involved in the invasion and metastasis of glioma cells via EMT. To the best of the authors' knowledge, the present study was the first to investigate the biological functions of CDK10 in glioma, and the first to report the involvement of CDK10 in EMT.

The zinc-finger transcription factor, Snail, is an essential factor for EMT (20-22). It has been demonstrated that Snail inhibits E-cadherin transcription by binding the E-box position that exists in the E-cadherin gene promoter (22). The inverse association between Snail and E-cadherin has been 


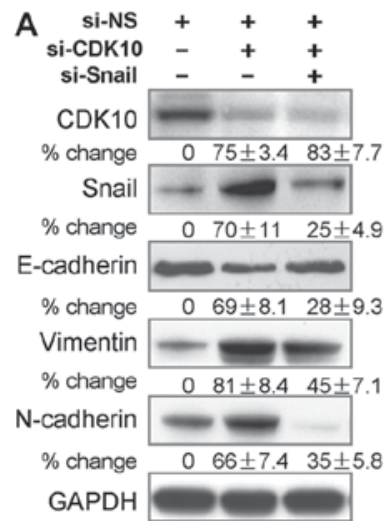

B

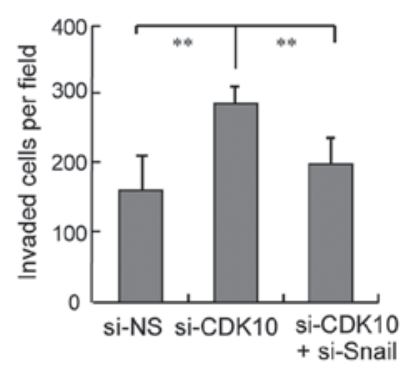

C

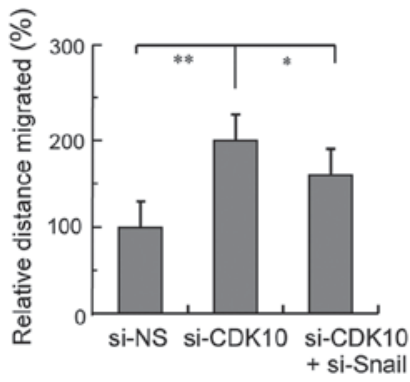

D

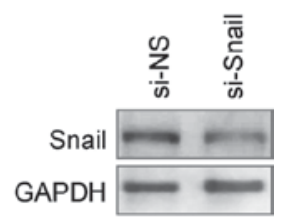

Figure 4. Snail-knockdown causes partial reversion of epithelial mesenchymal induced by CDK10-silencing. U251 cells were co-transfected with siRNAs targeting CDK10 and Snail. (A) Western blot analysis of the protein expression levels of epithelial and mesenchymal markers. (B) Cell invasion and (C) wound-healing assays revealed increased motility compared with control cells, while Snail-knockdown caused a significant reduction in the migratory ability of U251 cells with silenced CDK10. (D) Knockdown of Snail by siRNA in U251 cells was confirmed by western blot analysis. Each experiment was repeated $\geq 3$ times. "P $<0.05,{ }^{* *} \mathrm{P}<0.01$. si, small interfering; CDK, cyclin dependent kinase; E, epithelial; N, neural; si-NS, non-specific control siRNA; Snail, zinc finger protein SNAI1.

demonstrated in various types of cancer (20-22). In addition, it has been indicated that Snail expression is associated with World Health Organization-graded gliomas and that it may serve as a prognostic indicator (23). In the present study, CDK10 was demonstrated to regulate the expression level of Snail, although not Slug, ZEB1 or Twist. siRNA knockdown of Snail attenuated EMT-associated behavioral alterations in CDK10-silenced glioma cells and partially recovered the dysregulated expression pattern of EMT markers, suggesting an association between Snail and CDK10-regulated EMT in glioma cells. Previous studies have demonstrated that various other transcription factors, including ZEB2 and E47, regulate EMT during tumor metastasis (20-22). Future studies may examine whether Snail co-functions with other transcription factors to regulate CDK10-mediated EMT in glioma.

CDK10 has been demonstrated to be involved in endocrine therapy resistance in breast cancer through modulation of the protein c-ets- $2 / \mathrm{c}-\mathrm{RAF}$ proto-oncogene serine/threonine-protein kinase/mitogen activated protein kinase (MAPK) pathway (9). Previous research has demonstrated that activation of the MAPK pathway may function in EMT in multiple cancers (24). Therefore, it may be proposed that CDK10-silencing promotes EMT and metastatic progression by activating the MAPK/extracellular signal regulated kinase (ERK) pathway in glioma cells. Further investigation is required to confirm that the involvement of this signaling pathway in EMT activation is caused by loss of CDK10.

The tight junction protein occludin has been indicated to be involved in epithelial barrier modulation and cell adhesion, and downregulated occludin expression has been demonstrated to be associated with tumor aggressiveness and patient survival (25-27). The present study indicated no effect on occludin expression following knockdown or overexpression of CDK10. This observation was not unexpected since Slug, another master regulator of EMT, trans-activates the occludin gene promoter (28) and Slug protein expression was detected in the present study, regardless of CDK10 dysregulation. These results suggested that neither occludin nor Slug are required for CDK10-regulated EMT.

In conclusion, the present study suggested that CDK10 was able to inhibit glioma metastasis by inactivating Snail-mediated EMT. Due to its clinical application value, CDK10 may act as a molecular target for the prevention of glioma metastasis.

\section{Acknowledgements}

The authors would like to thank Ms. Jiongyu Chen (Oncological Research Laboratory, Cancer Hospital of Shantou University Medical College, Shantou, China) for technical assistance.

\section{Funding}

The present study was supported in part by the National Natural Science Foundation of China (grant no. 81760440).

\section{Availability of data and materials}

The dataset used and/or analyzed in the current study is available from the corresponding authors on reasonable request.

\section{Authors' contributions}

HL, JL and YY carried out experiments and analyzed the data. JL and YY conceived the study and designed the experiments.

\section{Ethics approval and consent to participate}

Not applicable.

\section{Consent for publication}

Not applicable.

\section{Conflicts of interests}

The authors declare that they have no competing interests. 


\section{References}

1. Armstrong TS, Wen PY, Gilbert MR and Schiff D: Management of treatment-associated toxicites of anti-angiogenic therapy in patients with brain tumors. Neuro Oncol 14: 1203-1214, 2012.

2. Ohgaki $\mathrm{H}$ and Kleihues P: Epidemiology and etiology of gliomas. Acta Neuropathol 109: 93-108, 2005.

3. Wen PY and Kesari S: Malignant gliomas in adults. N Engl J Med 359: 492-507, 2019.

4. Van Meir EG, Hadjipanayis CG, Norden AD, Shu HK, Wen PY and Olson JJ: Exciting new advances in neuro-oncology: The avenueto a cure for malignant glioma. CA Cancer J Clin 60: 166-193, 2010.

5. Nakada M, Nakada S, Demuth T, Tran NL, Hoelzinger DB and Berens ME: Molecular targets of glioma invasion. Cell Mol Life Sci 64: 458-478, 2007.

6. Graña X, Claudio PP, De Luca A, Sang N and Giordano A: PISSLRE, a human novel CDC2-related protein kinase. Oncogene 9: 2097-2103, 1994.

7. Brambilla R and Draetta G: Molecular cloning of PISSLRE, a novel putative member of the cdk family of protein serine/threonine kinases. Oncogene 9: 3037-3041, 1994.

8. Li S, MacLachlan TK, De Luca A, Claudio PP, Condorelli G and Giordano A: The cdc-2-related kinase, PISSLRE, is essential for cell growth and acts in G2 phase of the cell cycle. Cancer Res 55: 3992-3995, 1995.

9. Iorns E, Turner NC, Elliott R, Syed N, Garrone O, Gasco M, Tutt AN, Crook T, Lord CJ and Ashworth A: Identification of CDK10 as an important determinant of resistance to endocrine therapy for breast cancer. Cancer Cell 13: 91-104, 2008.

10. You Y, Yang W, Wang Z, Zhu H, Li H, Lin C and Ran Y: Promoter hypermethylation contributes to the frequent suppression of the CDK10 gene in human nasopharyngeal carcinomas. Cell Oncol (Dordr) 36: 323-331, 2013.

11. Yu JH, Zhong XY, Zhang WG, Wang ZD, Dong Q, Tai S, Li H and Cui YF: CDK10 functions as a tumor suppressor gene and regulates survivability of biliary tract cancer cells. Oncol Rep 27: 1266-1276, 2012.

12. Zhong XY, Xu XX, Yu JH, Jiang GX, Yu Y, Tai S, Wang ZD and Cui YF: Clinical and biological significance of Cdk10 in hepatocellular carcinoma. Gene 498: 68-74, 2012.

13. You Y, Li H, Qin X, Zhang Y, Song W, Ran Y and Gao F: Decreased CDK10 expression correlates with lymph node metastasis and predicts poor outcome in breast cancer patients-a short report. Cell Oncol 38: 485-491, 2015.

14. Hong CQ, Zhang F, You YJ, Qiu WL, Giuliano AE, Cui XJ, Zhang GJ and Cui YK: Elevated Clorf63 expression is correlated with CDK10 and predicts better outcome for advanced breast cancers: A retrospective study. BMC Cancer 15: 548, 2015.

15. You Y, Liu J, Wang Z, Zhang Y, Ran Y, Guo X, Liu H and Wang $\mathrm{H}$ : The enhancement of radiosensitivity in human esophageal squamous cell carcinoma cells by zoledronic acid and its potential mechanism. Cytotechnology 66: 17-25, 2014.
16. You Y, Yang W, Qin X, Wang F, Li H, Lin C, Li W, Gu C, Zhang Y and Ran Y: ECRG4 acts as a tumor suppressor and as a determinant of chemotherapy resistance in human nasopharyngeal carcinoma. Cell Oncol 38: 205-214, 2015.

17. Lin C, Xin S, Qin X, Li H, Lin L and You Y: Zoledronic acid suppresses metastasis of esophageal squamous cell carcinoma cells through upregulating the tight junction protein occludin. Cytotechnology 68: 1233-1241, 2016.

18. Lee KH, Ahn EJ, Oh SJ, Kim O, Joo YE, Bae JA, Yoon S, Ryu HH, Jung S, Kim KK, et al: KITENIN promotes glioma invasiveness and progression, associated with the induction of EMT and stemness markers. Oncotarget 6: 3240-3253, 2015.

19. Pang H, Zheng Y, Zhao Y, Xiu X and Wang J: miR-590-3p suppresses cancer cell migration, invasion and epithelial-mesenchymal transition in glioblastoma multiforme by targeting ZEB1 and ZEB2. Biochem Biophys Res Commun 468: 739-745, 2015.

20. Thiery JP, Acloque H, Huang RY and Nieto MA: Epithelial-mesenchymal transitions in development and disease. Cell 139: 871-890, 2009.

21. Martin TA, Goyal A, Watkins G and Jiang WG: Expression of the transcription factors snail, slug, and twist and their clinical significance in human breast cancer. Ann Surg Oncol 12: 488-496, 2005.

22. Nagaishi M, Paulus W, Brokinkel B, Vital A, Tanaka Y, Nakazato Y, Giangaspero $\mathrm{F}$ and Ohgaki $\mathrm{H}$ : Transcriptional factors for epithelial-mesenchymal transition are associated with mesenchymal differentiation in gliosarcoma. Brain Pathol 22: 670-676, 2012.

23. Myung J, Cho BK, Kim YS and Park SH: Snail and Cox-2 expressions are associated with WHO tumor grade and survival rate of patients with gliomas. Neuropathology 30: 224-231, 2010.

24. Xu T, Zhou M, Peng L, Kong S, Miao R, Shi Y, Sheng H and Li L: Upregulation of CD147 promotes cell invasion, epithelial-to-mesenchymal transition and activates MAPK/ERK signaling pathway in colorectal cancer. Int J Clin Exp Pathol 7: 7432-7441, 2014.

25. Kimura Y, Shiozaki H, Hirao M, Maeno Y, Doki Y, Inoue M, Monden T, Ando-Akatsuka Y, Furuse M, Tsukita S and Monden M: Expression of occludin, tight-junction-associated protein, in human digestive tract. Am J Pathol 151: 45-54, 1997.

26. Martin TA, Mansel RE and Jiang WG: Loss of occludin leads to the progression of human breast cancer. Int J Mol Med 26: 723-734, 2010.

27. Tobioka H, Isomura H, Kokai Y, Tokunaga Y, Yamaguchi J and Sawada N: Occludin expression decreases with the progression of human endometrial carcinoma. Hum Pathol 35: 159-164, 2004.

28. Wang Z, Wade P, Mandell KJ, Akyildiz A, Parkos CA, Mrsny RJ and Nusrat A: Raf 1 represses expression of the tight junction protein occludin via activation of the zinc-finger transcription factor slug. Oncogene 26: 1222-1230, 2007. 